Supplement of Biogeosciences, 18, 4185-4209, 2021

https://doi.org/10.5194/bg-18-4185-2021-supplement

(c) Author(s) 2021. CC BY 4.0 License.

(c) (1)

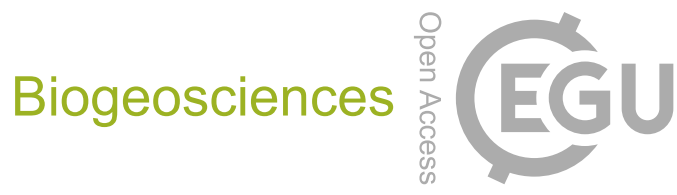

Supplement of

\title{
Wildfire history of the boreal forest of south-western Yakutia (Siberia) over the last two millennia documented by a lake-sediment charcoal record
}

\section{Ramesh Glückler et al.}

Correspondence to: Ramesh Glückler (ramesh.glueckler@awi.de) and Elisabeth Dietze (elisabeth.dietze@awi.de)

The copyright of individual parts of the supplement might differ from the article licence. 


\section{Wildfire history of the boreal forest of southwestern Yakutia (Siberia) over the last two millennia documented by a lake-sedimentary charcoal record}

\section{Supplement}

Overview of the different charcoal size classes (S1-S3) and morphotype classes (S4-S6) in both the classic and robust analysis approach, separated by vertical dashed lines representing the different phases of the fire regime.

For each figure: (a) Classic CHAR peak component (dark-grey bars = signal, light-grey bars = noise, dashed horizontal line = threshold). (b) SNI of the classic CHAR peak component after Kelly et al. (2011) (red horizontal line = SNI cutoff value of 3). (c) Classic CHAR sum (black line = interpolated CHAR, blue line = LOESS representing the CHAR background component, red vertical lines = fire episodes with $\mathrm{SNI}>3$, grey vertical lines = fire episodes with $\mathrm{SNI}<3$ ). (d) Robust CHAR background component. (e) Robust CHAR peak component (red areas = above-average values).

(f) Robust CHAR sum. For (d)-(f): black line = median, grey area = interquartile range.

S1: Size class $150-300 \mu \mathrm{m}$

S2: Size class $300-500 \mu \mathrm{m}$

S3: Size class $>500 \mu \mathrm{m}$

S4: Angular morphotypes (S, B, C)

S5: Elongated morphotypes ( $F, D, E)$

S6: Irregular morphotypes (M, P, X) 
S1: Size class $150-300 \mu \mathrm{m}$

Phase 1

Phase 2

Phase 3

Phase 4

$\ulcorner\quad 1.0-$ (a) Classic CHAR peak

ֻับ

\begin{tabular}{ll}
\hline & 0.6 \\
\hline & 0.4
\end{tabular}

\& 0.2

$\frac{0}{\frac{0}{*}}$

0.0

$-+-$

$-0.4$

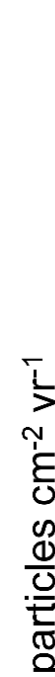

(b) SNI

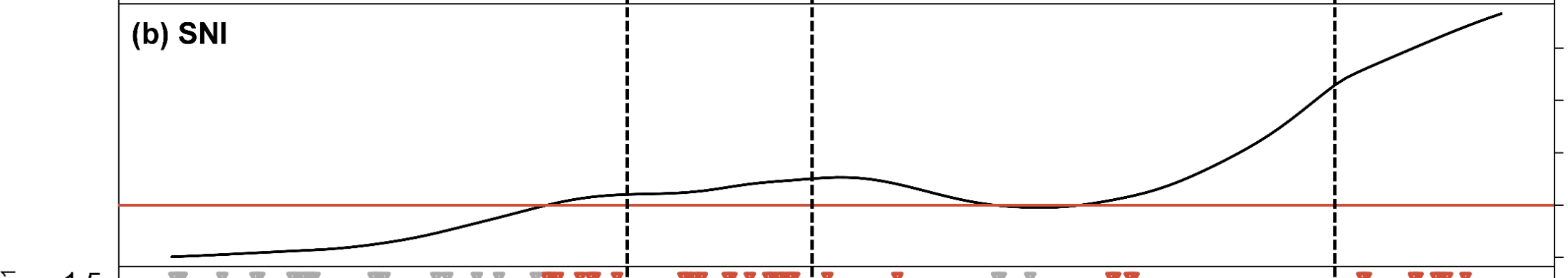

4.5

4.0

3.5

3.0

$\varlimsup_{7} \quad 1.5-$ (c) Classic CHAR

है 1.0

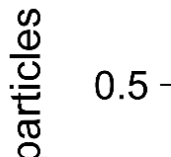

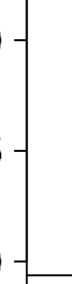

(n)

(d) Robust CHAR background

$\begin{array}{lr}0 & 1.0 \\ \stackrel{0}{0} & 0.5 \\ \mathcal{N} & 0.0 \\ -0.5 \\ -1.0 \\ -1.5 \\ -2.0\end{array}$

(e) Robust CHAR peak
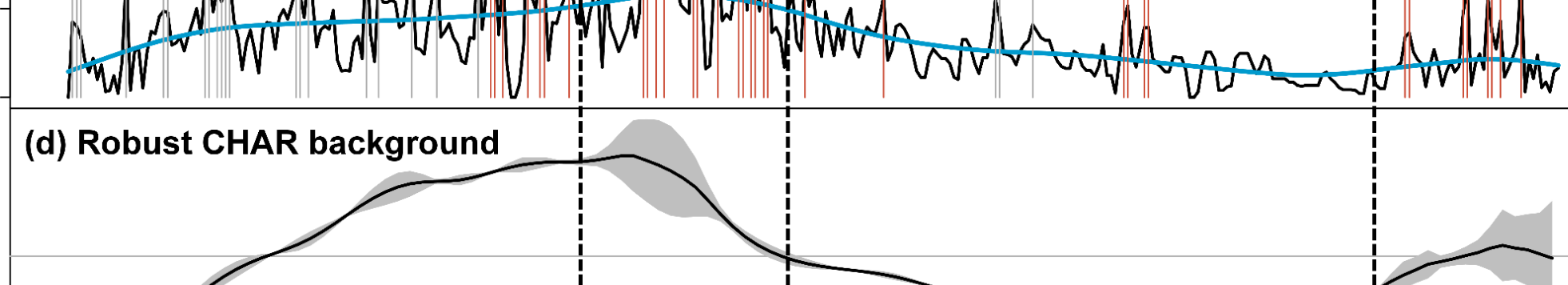

5

0.0
-0.5

$-1.0$

$-1.5$

(f) Robust CHAR

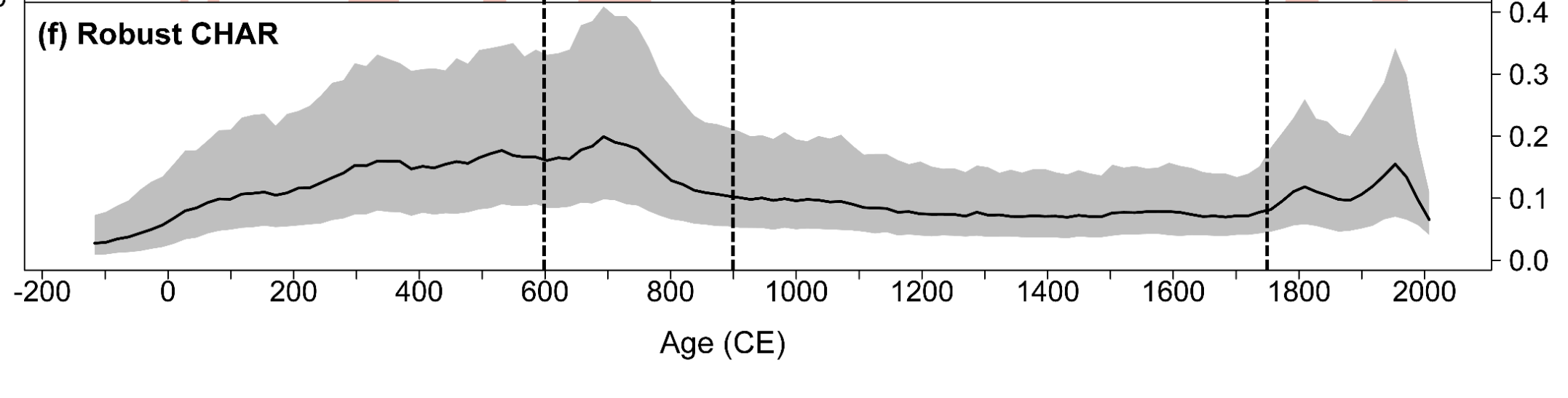


S2: Size class $300-500 \mu \mathrm{m}$

Phase 1

Phase 2

Phase 3

Phase 4

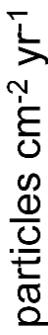

$\quad 0.4-$ (a) Classic CHAR peak

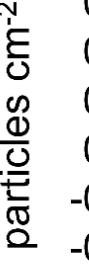

$-0.3$

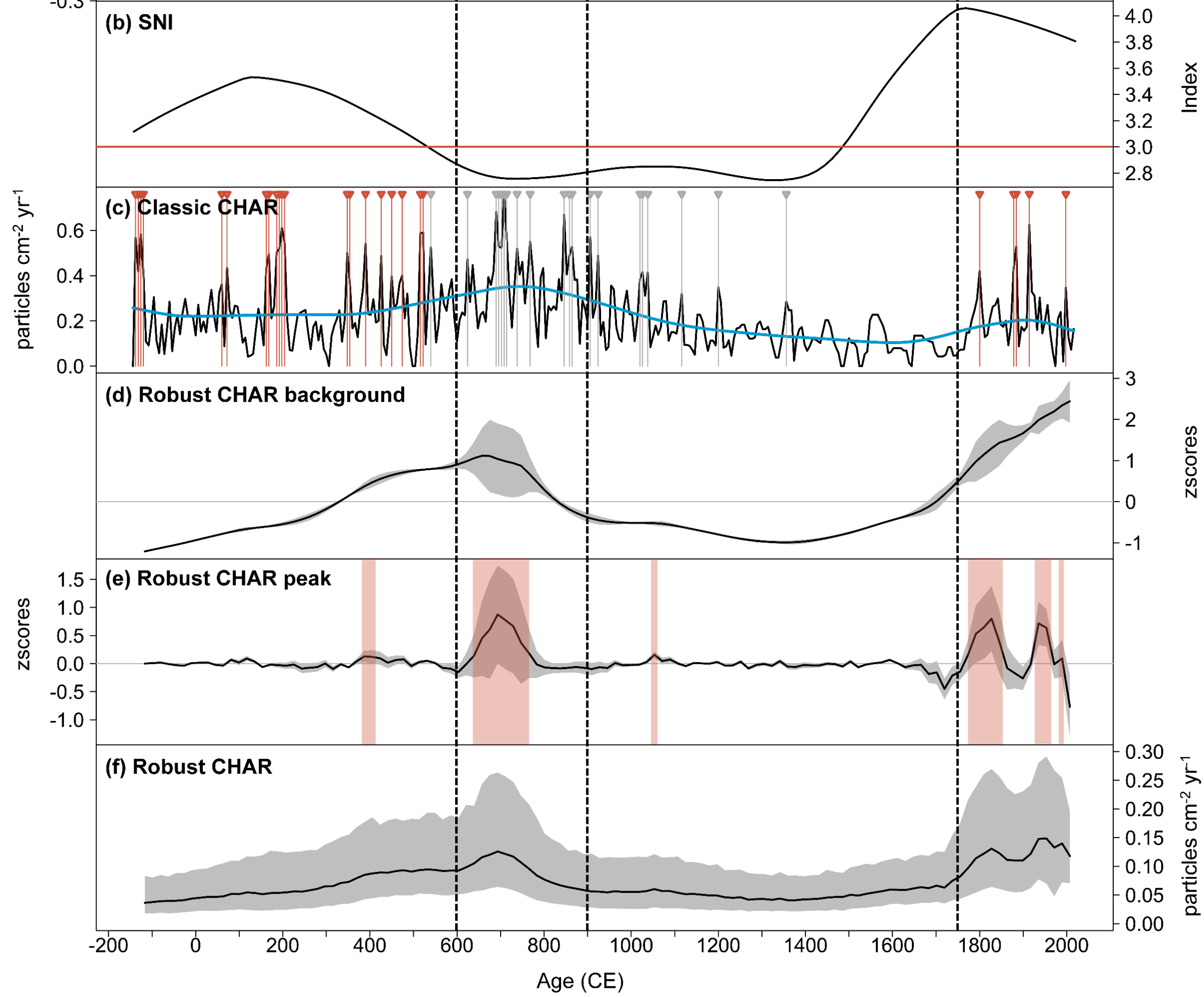


S3: Size class $>500 \mu \mathrm{m}$

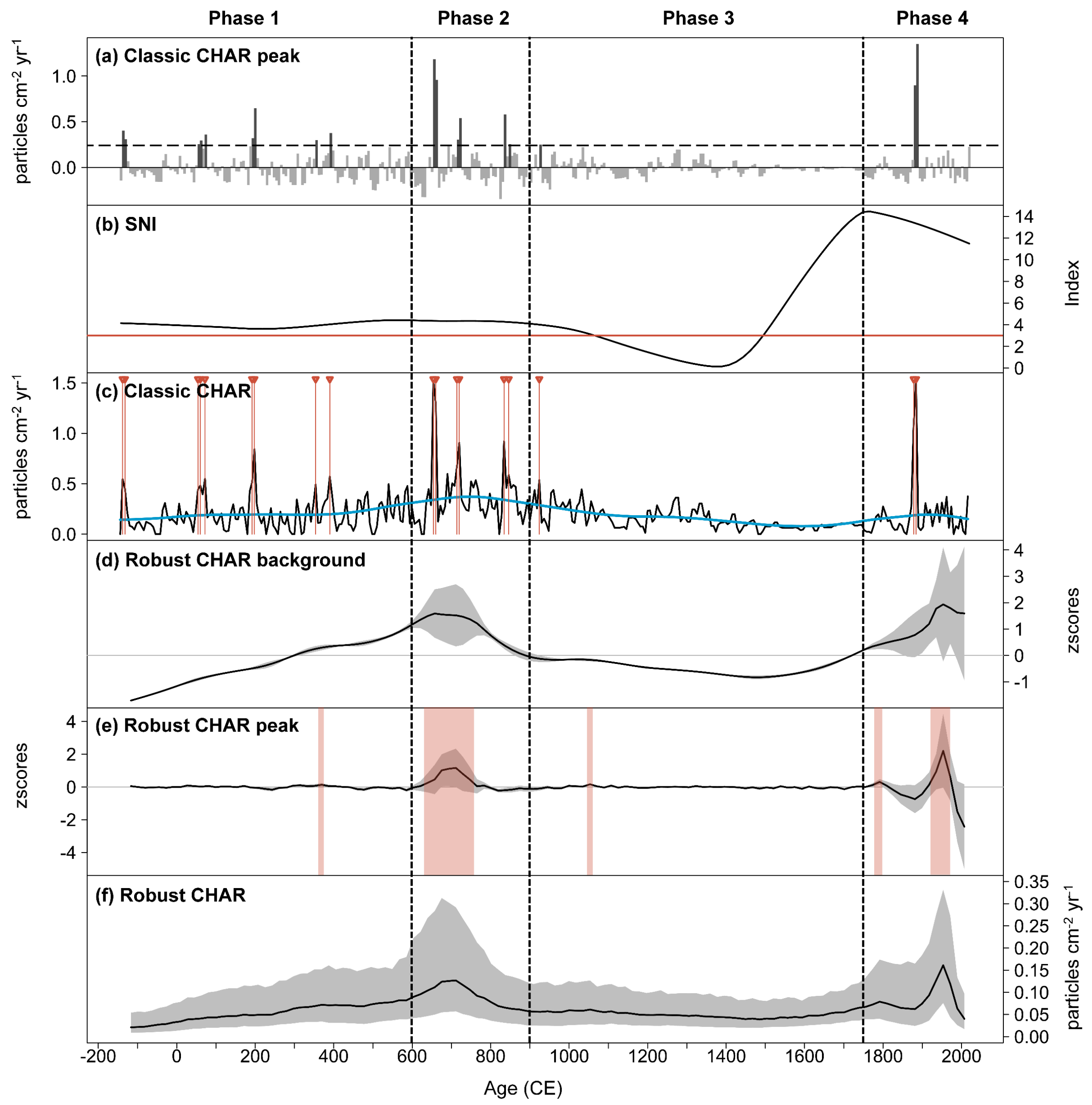


S4: Angular morphotypes

Phase 1

Phase 2

Phase 3

Phase 4

$\frac{1}{2}$
$\frac{1}{0}$
0
$\frac{1}{0}$
.0
$\frac{2}{2}$
$\frac{2}{2}$

$\succsim \quad 0.6-$ (a) Classic CHAR peak

है 0.4

品

0.
0.
-0.

(b) SNI

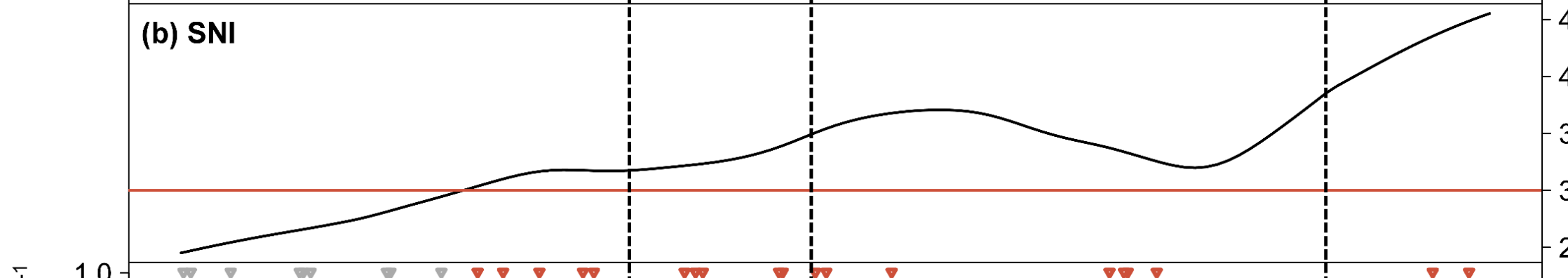

4.5

4.0

3.5 으

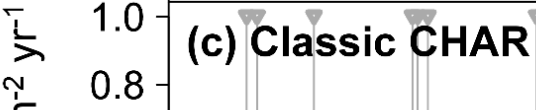

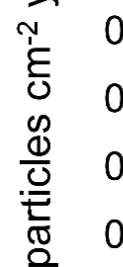

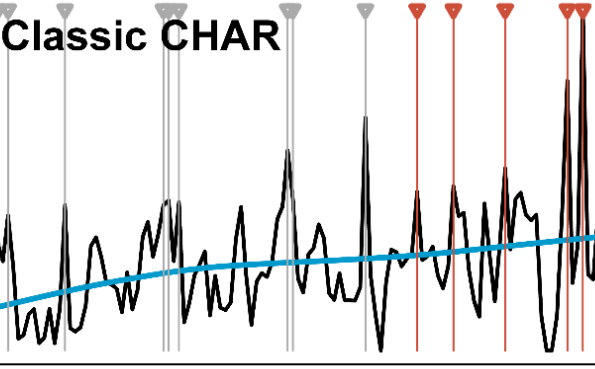

(d) Robust CHAR background

(e) Robust CHAR peak

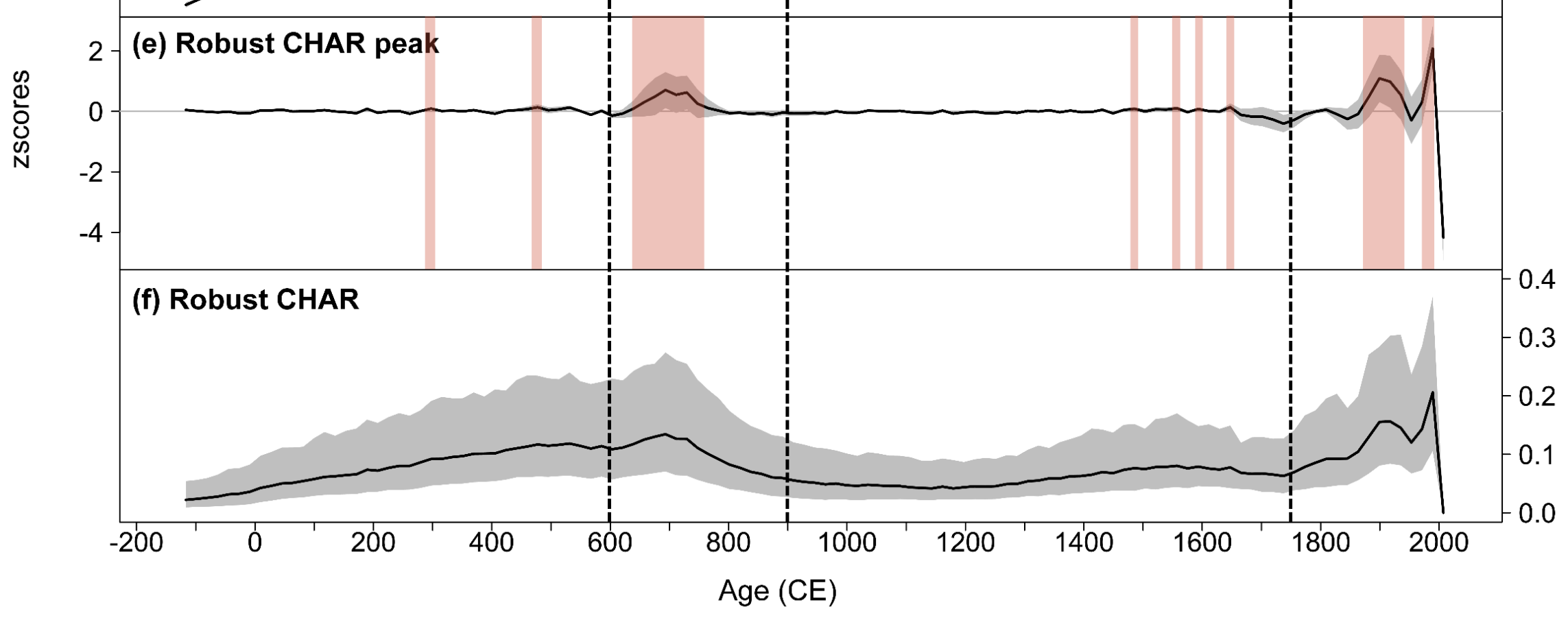

$\frac{1}{2}$
$\frac{1}{0}$
$\frac{1}{0}$
$\frac{1}{0}$
$\frac{0}{2}$
$\frac{1}{0}$ 


\section{S5: Elongated morphotypes}

Phase 1

Phase 2

Phase 3

Phase 4

ָัָ

(a) Classic CHAR peak

0.8

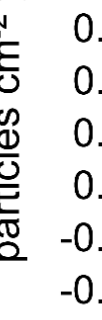

0.4
0.2
0.0

$-0.2$

(b) SNI

בิ

$\left.\begin{array}{ll}1.4 \\ 1.2 \\ 1.0 \\ 0\end{array}\right]$ (c)

(c) Classic CHAR

(d) Robust CHAR background

(e) Robust CHAR peak

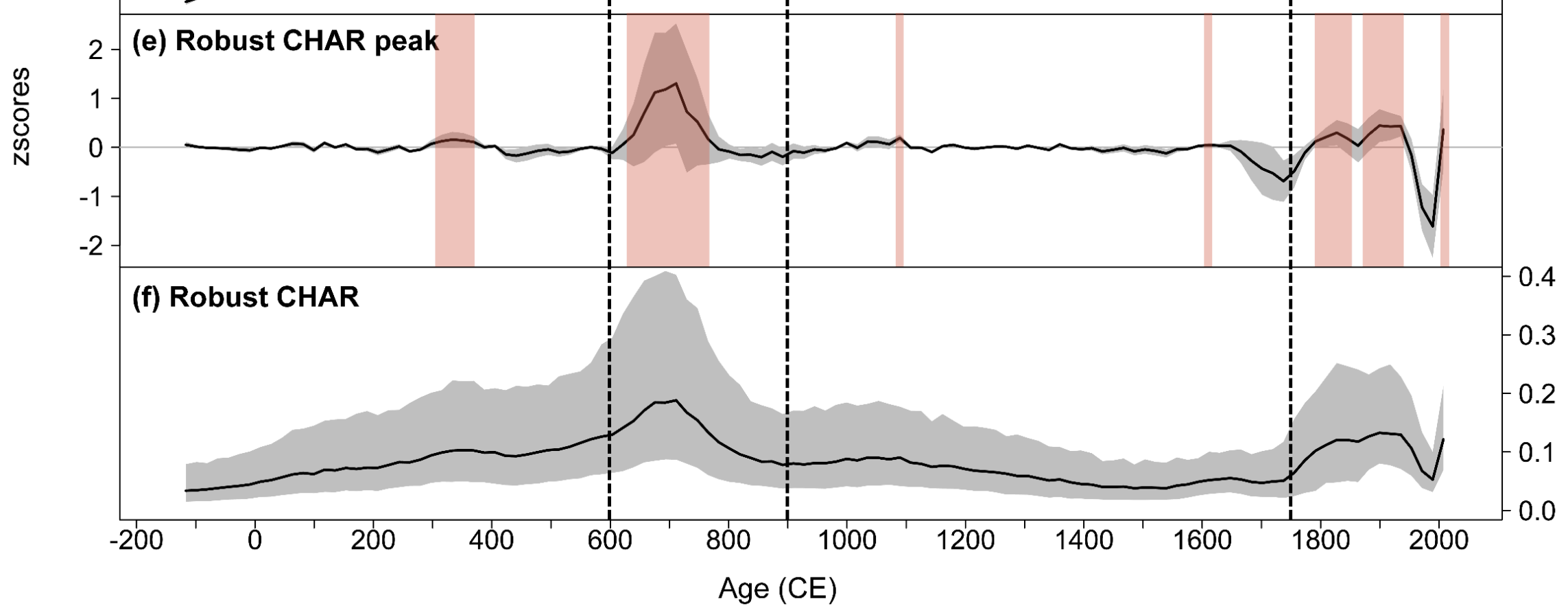

ֻู 
S6: Irregular morphotypes

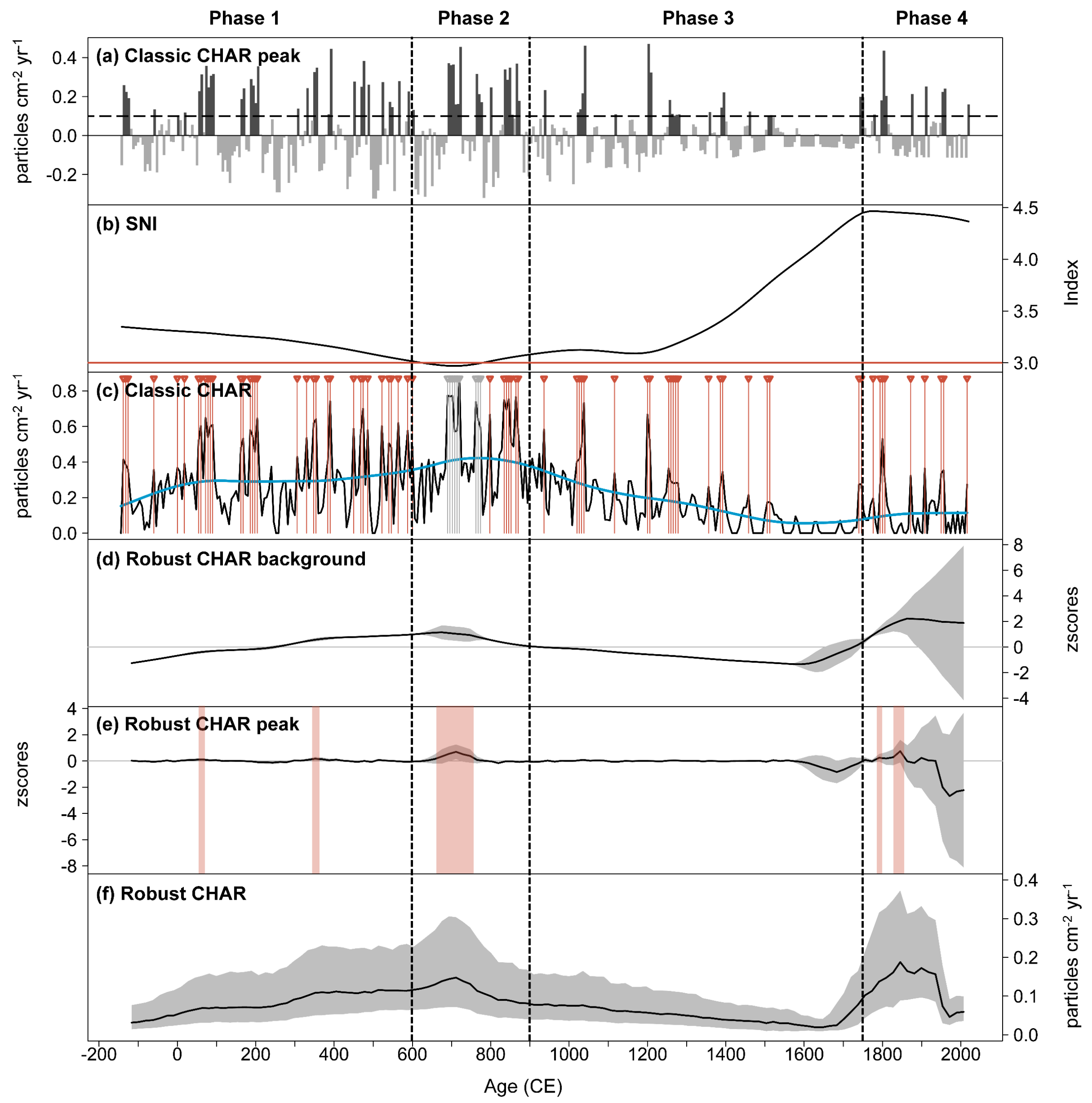

